Conference abstract PMS18

\title{
Simulation of Particles with Different Coefficient of Restitution in Fluid Bed Coater
}

\author{
R. ŠIBANC, R. DREU, S. SRČIČ
}

Faculty of Pharmacy, University of Ljubljana, Ljubljana, Slovenia

E-mail: rok.sibanc@ffa.uni-lj.si (R. Šibanc)

Sci Pharm. 2010; 78: 645

doi:10.3797/scipharm.cespt.8.PMS18

Particle coating using fluid bed technology such as Wurster chamber is a common process in pharmaceutical technology. Coatings are used to achieve variety of functions, such as: taste masking, controlled release, increase of stability and others. Coating uniformity is one of the most important parameters of coated particles and can play an important role in the release of the active ingredient and is a result of number of process settings and material properties $[1,2]$. One of the material parameter that effects particle movement and consequent coating is coefficient of restitution, which is defined as the ratio of particle velocity before and after the impact [3].

We have analysed the effect of coefficient of restitution on particle distribution and their velocity in the Wurster process chamber. Simulations were performed on a $2 \mathrm{D}$ axisymetric model using Ansys Fluent. Euler-Euler approach was used for simulation of gas and solid phase. The particle size was set at $1 \mathrm{~mm}$ and the coefficient of restitution was set from 0,1 to 1,0 in 0,1 steps. All other process parameters such as fluidizing and atomizing air flow were kept constant. $10 \mathrm{~s}$ of two-phase flow was calculated in each simulation using timestep of $2.5^{*} 10^{-5} \mathrm{~s}$, starting from the same initial condition.

The analysis of the simulation has established that in most cases higher values of coefficient of restitution increase the particle volume fraction in the coating region of the chamber. The lowest value of volume fraction of particles in the coating region was $6.82 \%$ and the highest was $9.41 \%$. It was established that coefficient of restitution has effect on particle velocity and flow rate through the coating region, however far less pronounced as in the case of volume fraction, which is one of the main factors that affects uniformity of coating. Therefore, according to our findings elastic properties of the particles can affect the outcome of the coating process i. e. coating functionality, even when using the same process chamber and process parameters.

[1] Cheng X, Turton R. The prediction of variability occurring in fluidized bed coating equipment. I. The measurement of particle circulation rates in a bottom-spray fluidized bed coater. Pharm Dev Technol. 2000; 5: 311-322. doi:10.1081/pdt-100100546

[2] Dreu R, Perpar B, Zun I, Srcic S. Influence of hydrodynamic conditions in Wurster chamber on uniformity of the pellet film coating thickness. Eur J Pharm Sci. 2005; 25: S92-S94.

doi:10.1016/j.ejps.2005.04.007

[3] Du W, Bao X, Xu J, Wei W. Computational fluid dynamics (CFD) modeling of spouted bed: Influence of frictional stress, maximum packing limit and coefficient of restitution of particles. Chem Eng Sci.

2006; 61: 4558-4570. doi:10.1016/j.ces.2006.02.028 